\title{
STEM Strain Measurement From a Stream of Diffraction Patterns Recorded on a Pixel-Free Delay-Line Detector.
}

\author{
Knut Müller-Caspary ${ }^{1}$, Andreas Oelsner ${ }^{2}$, Pavel Potapov ${ }^{3}$ \\ 1. IFP, Universität Bremen, Otto-Hahn-Allee 1, 28359 Bremen, Germany. \\ 2. Surface Concept GmbH, Am Sägewerk 23a, 55124 Mainz, Germany. \\ ${ }^{3 .}$ GLOBALFOUNDRIES Dresden Module 1, Wilschdorfer Landstr. 101, 01109 Dresden, Germany.
}

Recent progress in nano-beam electron diffraction (NBED), ptychography and differential phase contrast microscopy [1] is based on the acquisition of a four-dimensional data $\operatorname{set} I\left(x_{p}, y_{p}, x, y\right)$ with $\left(\mathrm{x}_{\mathrm{p}}, \mathrm{y}_{\mathrm{p}}\right)$ the position of the STEM probe, $(\mathrm{x}, \mathrm{y})$ a coordinate in the recorded image/diffraction pattern and intensity I. Since the speed of conventional charge-coupled devices is limited, a major challenge is the development of fast detectors.

Here we present pilot experiments with a delay-line detector (DLD, [2]) mounted on an FEI Titan facility. As illustrated in Fig.1 the top of the DLD consists of a microchannel plate (MCP) stack that causes a cascade of secondary electrons for each $300 \mathrm{keV}$ electron impinging on the detector. The heart of the DLD are 2 meandering wires shown in blue and red in which each cascade causes electrical pulses travelling towards the ends of the wires. Depending on the incident position of the electron a characteristic time delay between the arrivals of the 2 conjunct pulses at the ends of a wire is measured with high accuracy, giving the coordinate of incidence perpendicular to the meander. By crossing two such delay-lines the point $(x, y)$ and time $t$ of incidence can be detected. Thus the DLD allows for both the recording of a continuous stream of single electron events processed by a time-to-digital converter (TDC) with a time precision in the picosecond range and the in-situ integration of the signal over a certain frame time to obtain conventional images. Note that no pixel raster is involved here. The two modes of operation are illustrated in the right part of Fig. 1. To characterise the DLD, we determined the quantum efficiency (QE) as a function of the incident intensity as shown in Fig. 2. The QE takes values between $50 \%$ for $0.5 \times 10^{6}$ and $22 \%$ for $17 \times 10^{6}$ electrons/second for a homogeneous illumination of the detector. Moreover, the modulation transfer function was determined to 0.1 at Nyquist frequency.

Finally, we present strain measurements. An example is shown in Fig. 3 for a GeSi/Si MOSFET for the [001] and [110] direction at a STEM raster of 100x100 pixels. In particular we observe 2 strain regimes inside the GeSi stressors owing to different Ge contents of $25 \%$ and $37 \%$, as has been verified by quantitative STEM Z-contrast evaluations. In Fig.3a, strain $\varepsilon_{y y}$ in [001] direction was measured from the position of the 004 reflection [3] whereas the 220 reflection was used for the lateral strain measurement of $\varepsilon_{\mathrm{xx}}$ in Fig.3b. In between the stressors, the gate channel exhibits compressive strain of up to $3 \%$ laterally and an expansion below 1\% along [001]. Dwell times between 40 and 5ms have been used, corresponding to an acquisition of 10,000 diffraction patterns in 6.5 and $0.8 \mathrm{~min}$, respectively.

\section{References}

[1] K. Müller et al., Nature Communications 5 (2014), 5653.

[2] K. Müller-Caspary, A. Oelsner, P. Potapov, Applied Physics Letters 107 (2015), 072110.

[3] K. Müller and A. Rosenauer et al., Microscopy and Microanalysis 18 (2012), 995.

[4] Funding: Deutsche Forschungsgemeinschaft (DFG) under contract MU3660/1-1. 


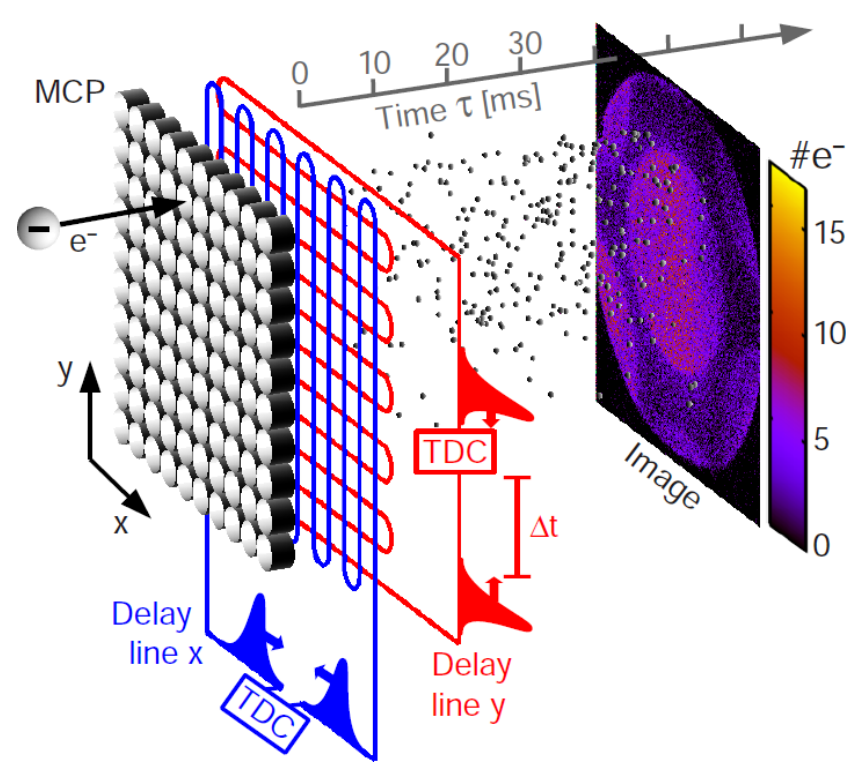

Figure 1. Schematic to illustrate the functional principle of the delay-line detector. An incident electron causes a cascade in the microchannel plate (MCP) stack, leading to pulses in the meandering delay-lines which are processed by a time-to-digital (TDC) converter.

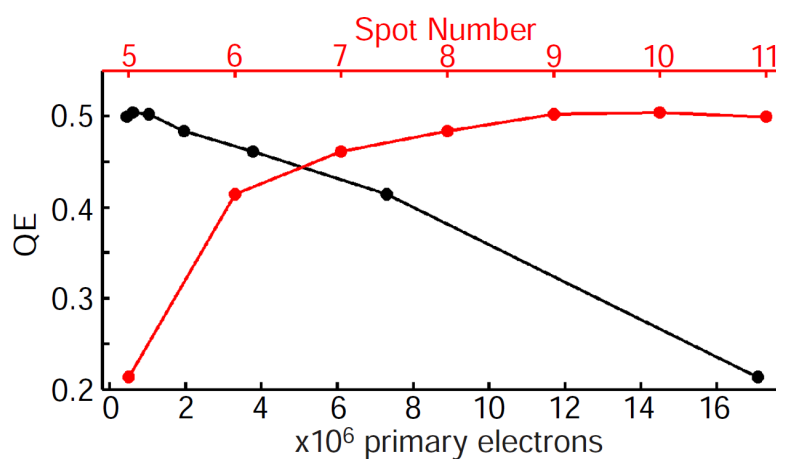

Figure 2. Quantum efficiency of the DLD as a function of the incident dose (black) (per second). Red: dependence on the Spot Number in an FEI Titan operated at 300kV in HRSTEM mode.

(a)

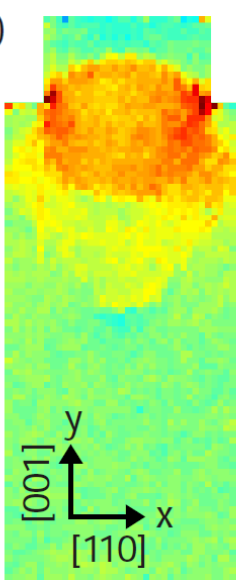

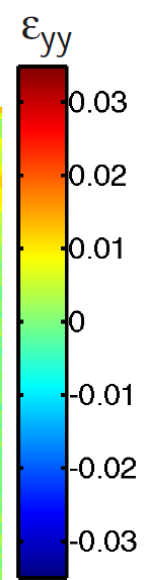

(b)

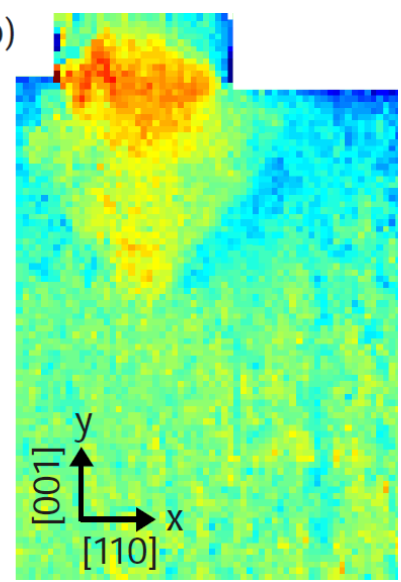

Figure 3. Maps for (a) vertical and (b) lateral strain in a Si-based MOSFET test structure measured from the 004 and 220 reflection and 40ms dwell times, respectively. The GeSi stressors cause a compression of up to 3\% along [110] and an expansion below 1\% along [001] in the gate region. 